\title{
Central $\mathrm{Mg}_{2}$ indices for early-type galaxies ${ }^{\star, \star \star}$
}

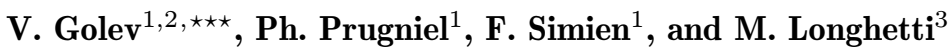 \\ 1 CRAL-Observatoire de Lyon, CNRS UMR 142, F-69561 St-Genis-Laval Cedex, France \\ 2 Department of Astronomy and Astronomical Observatory, University of Sofia, P.O. Box 36, BG-1504 Sofia, Bulgaria \\ 3 Institut d'Astrophysique de Paris, 98 bis boulevard Arago, F-75014 Paris, France
}

Received June 18, 1998; accepted February 1, 1999

\begin{abstract}
We present 210 new measurements of the central absorption line-strength $\mathrm{Mg}_{2}$ index for 87 early-type galaxies drawn from the Prugniel \& Simien (1996) sample. 28 galaxies were not observed before. The results are compared to measurements published previously as available in HYPERCAT, and rescaled to the Lick system. The mean individual internal error on these measurements is $0.009 \pm 0.003$ and the mean external error is $0{ }^{\mathrm{m}} 012 \pm 0{ }^{\mathrm{m}} 002$ for this series of measurements.
\end{abstract}

Key words: galaxies: elliptical and lenticular, $\mathrm{cD}$ galaxies: fundamental parameters - galaxies: stellar content

\section{Introduction}

The existence of the the Fundamental Plane (FP) of earlytype galaxies (Djorgovski \& Davies 1987) results from very simple scaling relations (de Carvalho \& Djorgovski 1989). The equilibrium status of galaxies (Virial Theorem) can be written (Prugniel \& Simien 1997):

$k_{\mathrm{K}} \sigma_{0}^{2}=(2 \pi)^{-1} k_{\mathrm{S}}(\mathcal{M} / L) L r_{\mathrm{e}}^{-1}$,

where $L, r_{\mathrm{e}}$ and $\sigma_{0}$ are respectively the luminosity, the effective radius, and the central velocity dispersion. $k_{\mathrm{S}}, k_{\mathrm{K}}$, and $\mathcal{M} / L$ are the three functions entering in the relations scaling respectively the gravitational energy, the kinetic energy, and the mass of galaxies.

The FP equation has the simple form

$k_{\mathrm{S}} k_{\mathrm{K}} \mathcal{M} / L=L^{\beta}$

Send offprint requests to: Ph. Prugniel (prugniel@galaxies. univ-lyon1.fr).

* Based on observations collected at the Observatoire de Haute-Provence.

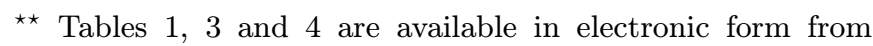
the CDS, Strasbourg (via anonymous ftp to 130.79.128.5).

Tables 1 and 3 are available from CDS only.

$\star \star \star$ E-mail: valgol@phys.uni-sofia.bg with $\beta$ ranging from 0.2 to 0.1 when going from the $B$ to $K$-color band (e.g. Djorgovski \& Santiago 1993). This weak dependence on the luminosity (the so called "tilt" of the FP - see Renzini \& Ciotti 1993) implies the quasilinearity of the scaling relations, i.e., the kinetic energy must be almost proportional to $\sigma_{0}$, the mass to the luminosity, and so on.

Studying the residuals from the FP as a function of other parameters gives insights into the details of the scaling relations. For instance, Prugniel \& Simien (1994) expressed the contribution of the rotational support to the total kinetic energy as $k_{\mathrm{K}}=1+0.81\left(V_{\max } / \sigma_{0}\right)^{2}$. The nonlinearity of the scaling of the gravitational energy (nonhomology of the spatial structure) has also been found by Busarello et al. (1996) (see also Prugniel \& Simien 1996). Combined, these two effects account for roughly half the tilt of the FP in the $B$-band. The scaling of the mass is more complicated since it combines the characteristics of the stellar population to those of the dark halo. However, the correlation between the residuals to the FP and the color or $\mathrm{Mg}_{2}$ (Prugniel \& Simien 1996) indicates a strong contribution of the diversity of the stellar populations to the spread around the FP (Guzmán et al. 1992; Jørgensen et al. 1996). Because of the color-magnitude (and $\mathrm{Mg}_{2}{ }^{-}$ magnitude) relations, this accounts for the rest of the tilt of the FP in the $B$-band. The smaller tilt observed in the near IR (Recillas-Cruz et al. 1990; Scodeggio et al. 1998) confirms this. Then, unless some other unidentified phenomena add other compensatory tilts, the fraction of dark matter can be considered constant in the region probed by this analysis (i.e. within $1-2 r_{\mathrm{e}}$ ).

These effects are of interest for the understanding of the physics of galaxies, but are also important when dealing with large-scale streaming, using the FP as a distance indicator (Dressler et al. 1987), since they potentially result in spurious peculiar velocities (e.g. Gregg 1992).

In order to go deeper in the analysis of the connection between the residuals to the $\mathrm{FP}$ and the characteristics 
of the stellar population we are continuing observational programs.

In the present paper we measure the central $\mathrm{Mg}_{2}$-index for the 87 galaxies already accumulated in our library. This material consists in medium resolution spectra $(3.2 \AA$ FWHM), and relevant details on it are given in Sect. 2. In Sect. 3, we describe the measurements method and error analysis and present their reduction to the homogeneous system defined in Golev \& Prugniel (1998). A comparison with other series of measurements (Sect. 4), taken from the HYPERCAT database ${ }^{1}$, ascertains the level of reliability of our data and measurement procedure.

\section{Sample and observations}

The current sample has previously been selected for kinematical studies. It is composed of 87 early-type galaxies (S0s and ellipticals between E0 and E4). Kinematical parameters were published in a series of five papers (Prugniel \& Simien 1994; Simien \& Prugniel 1997a, 1997b, 1997c, 1998 , hereafter collectively referred to as SP): the central velocity dispersion $\sigma_{0}$, the maximum rotational velocity $V_{\max }$ and, for some galaxies, the radial profile $\sigma(r)$ of the velocity dispersion, together with the rotation curve $V(r)$ are given in these papers. Although the sample is not complete in terms of limiting apparent or absolute magnitude, the galaxies of the sample span a wide range of intrinsic luminosity $\left(-22^{\mathrm{m}} 0 \lesssim M_{B} \lesssim-17^{\mathrm{m}} 5\right)$, of central velocity dispersion $\left(70 \lesssim \sigma_{0} \lesssim 330 \mathrm{~km} \mathrm{~s}^{-1}\right)$, and, as a consequence, of metallicity. The observations were made at the $1.93 \mathrm{~m}$ telescope of the Observatoire de Haute-Provence equipped with the $C A R E L E C$ long-slit spectrograph ${ }^{2}$ (Prugniel et al. 1992). The detector was a Tektronix CCD with $512 \times 512$ pixels of $27 \mu \mathrm{m}$ each. The slit width projected on the sky was 2.2 arcsec. CARELEC transforms the focal ratio from $\mathrm{f} / 15.5$ at the Cassegrain focus to a value six times faster, providing a scale of $1.1 \operatorname{arcsec} \mathrm{px}^{-1}$ in the spatial direction along the slit, and a FWHM spectoscopic resolution of $3.2 \AA$ (or $1.8 \mathrm{px}$ ) corresponding to $1.8 \AA \mathrm{px}^{-1}$. The selected setup with a reciprocal dispersion of $66 \AA \mathrm{mm}^{-1}$ is always centered on the $\mathrm{Mg} b \lambda 5175 \AA$ triplet, and it provides nearly $900 \AA$ of spectral coverage around it.

During seven observing runs in the period 1993 - 1995, a total of 210 spectra of early-type galaxies have been collected. Typically, two 45-min exposures were obtained for each galaxy. For comparison purposes, 17 galaxies of the sample were observed in more than one run. All details about the runs and the logs of observations can be found

\footnotetext{
1 The database HYPERCAT is developed and maintained at the Observatoire de Lyon (http://www-obs.univlyon1.fr/hypercat/).

${ }^{2}$ Details on the CARELEC long-slit spectrograph and its detectors, as well as the information about the $1.93 \mathrm{~m}$ telescope itself, are available on the WWW at http://www.obs-hp.fr
}

in SP. At least two template stars of spectral type in the range G8-III to K3-III were observed at the beginning of each night.

\section{Data reduction}

\subsection{Measurements and errors}

We have used the wavelength-calibrated and skysubtracted spectra resulting from the kinematical reduction presented in SP. In order to extract the central $\mathrm{Mg}_{2}$-indices we have integrated the signal in the aperture of $2.2 \times 5.5$ arcsec.

The flux calibration has been performed using spectra of the kinematical template stars common with the KPNO Coudé Feed Spectrophotometric Library (The Jones library - see Leitherer et al. 1996). The spectra in this library are flux-calibrated and can be regarded as "approximately spectrophotometric" (Worthey \& Ottaviani 1997). Seven late-type stars observed by us could be used for that purpose, totalling 11 spectra. The wavelength response of the system (atmosphere + telescope + spectrograph + CCD) was modelled by a second degree polynomial.

This small number of flux calibrators does not allow to perform a monitoring of the change in the atmospheric absorption, hence we determined the error due to the change in the flux calibration by comparing the $\mathrm{Mg}_{2}$-values obtained with the 11 different response functions. Assuming that the distribution of these 11 calibrations is representative of changes of the flux calibration during the whole runs, we found the corresponding error on $\mathrm{Mg}_{2}$ to be $\lesssim 0.004$.

Recently Worthey \& Ottaviani (1997) provided an elaborate mapping of the Lick system resolution which is about $8.4 \AA$ at $5300 \AA$. Our spectra were transformed to this resolution by convolving them with an appropriate gaussian. The raw values of $\mathrm{Mg}_{2}$-indices, $\left(\mathrm{Mg}_{2}\right)_{\mathrm{obs}}$, were measured according to the Lick definition (see Worthey et al. 1994):

$$
\left(\mathrm{Mg}_{2}\right)_{\mathrm{obs}}=-2.5 \log \left[\left(\frac{1}{\lambda_{2}-\lambda_{1}}\right) \int_{\lambda_{1}}^{\lambda_{2}} \frac{F_{\mathrm{I} \lambda}}{F_{\mathrm{C} \lambda}} \mathrm{d} \lambda\right]
$$

where $F_{\mathrm{I} \lambda}$ is the flux (in ergs $\mathrm{s}^{-1} \mathrm{~cm}^{-2} \AA^{-1}$ ) in the $\mathrm{Mg} \mathrm{H}$ and $\mathrm{Mg} b$ which are located at $\lambda \lambda 5154.125-$ $5196.625 \AA$. Here the local continuum, $F_{\mathrm{C} \lambda}$, represents the run of flux defined by a line connecting the flux levels at midpoints of the corresponding blue and red "continuum" bandpasses (which are actually pseudocontinua) at $\lambda \lambda 4895.125-4957.625 \AA$ and $\lambda \lambda 5301.125-5366.125 \AA$.

For each individual spectrum the $\mathrm{Mg}_{2}$-index error was first estimated as described in Cardiel et al. (1998), then the flux-calibration error was added quadratically to the obtained value.

We performed a series of tests to determine the sensitivity of our measurements to different sources of errors. 
The dominant uncertainty comes from the photon noise (typically $\sim 0 \mathrm{~m} 007$ ). The uncertainty on the flux calibration and on the sky subtraction come after (the overall effect of both these sources of errors is $\lesssim 0$. 006 ).

\subsection{Velocity dispersion correction}

Using the tabulation in Longhetti et al. (1998), we have adopted the following formula for the velocity dispersion correction:

$\left(\mathrm{Mg}_{2}\right)_{\sigma=0}=\left(\mathrm{Mg}_{2}\right)_{\mathrm{obs}}+0.001 \log \sigma$.

This correction (typically $\sim 0.002$ ) is in general well within the errors, but we applied it because it may bias the measurements in a systematic way.

\subsection{Aperture correction}

Because galaxies show radial gradients for the $\mathrm{Mg}_{2}$-index, this index must be corrected for the effect of the increasing projected aperture size in the more distant galaxies which weakens their indices. The aperture correction of the observed index $\left(\mathrm{Mg}_{2}\right)_{\text {obs }}$ was performed as it is proposed by Jørgensen et al. (1995) consistently with the procedures described in Golev \& Prugniel (1998) catalogue. The adopted normalized aperture is equivalent to an angular diameter of 3.4 arcsec for the distance to the Coma cluster. The distances were estimated as in Golev \& Prugniel (1998).

The mean aperture correction added to the raw measurements is $-0 \mathrm{~m} 016$ with an $\mathrm{rms}$ of 0.012 . The negative mean correction reflects the fact that our observations sample a physical aperture smaller than the normalisation aperture adopted in HYPERCAT (Golev \& Prugniel 1998). Inverting Eq. (3) from Golev \& Prugniel tells that in average the apertures used in our observations are 0.4 the normalisation radius.

The $\mathrm{Mg}_{2}$-index measurements for the 210 individual spectra are presented in Table 1 (in electronic form only). This table gives the individual velocity-dispersioncorrected and aperture-corrected measurements. The distances (in $\mathrm{km} \mathrm{s}^{-1}$ ) used to compute the aperture correction can be found in the electronic version of Table 4 . The mean internal error of individual measurements is 0. $0093 \pm 0$. 0032 which is typical of the values collected in HYPERCAT.

\section{Comparison with HYPERCAT}

In Golev \& Prugniel (1998) we have presented a catalogue of published absorption-line $\mathrm{Mg}_{2}$ indices of galaxies and globular clusters. This catalogue is maintained up-to-date in the HYPERCAT database and gathers more than 4000
$\mathrm{Mg}_{2}$-index measurements for $\sim 1500$ objects from $69 \mathrm{ob}-$ servational datasets reported in 41 different publications. Below we have used this database for the sake of internal and external comparison of our measurements.

\subsection{Internal comparisons}

During each observing run, we observed between 10 and 30 galaxies. In most cases, each observation consisted in two spectra with the same position angle interleaved with a calibration-lamp spectrum (needed for the kinematical analysis to control the possible flexures in the instrument). Two to five galaxies in each run were repeated in another run. This allowed two types of internal comparison: (1) between two consecutive spectra, and (2) between observation repeated during different observing runs. The relevant statistics of these comparisons is summarized in Table 2.

The results of these comparisons performed on the corrected measurements are:

- The rms difference between two consecutive observations of the same galaxy is $0.000 \pm 0.0096$. The only significant differences allowed to reject discrepant measurements affected by badly placed spikes due to cosmic-rays.

- The internal comparison did not reveal any evidence for significant zero-point difference (greater than 0.005) between the observing runs. But the relevance of this diagnostics is limited by the small number of comparison points (see below the external comparisons).

The internal comparison between the repeated measurements of the same galaxies reveals a mean error of 0.008 (the same order of magnitude as the total mean internal error of 0.0093 ), in agreement with the analysis of the error sources in the previous section.

\subsection{External comparisons}

Most of the galaxies in our sample were previously measured, in particular by the Lick group (Davies et al. 1987 hereafter LICK, and Faber et al. 1989 - hereafter 7Sam). Recently the full Lick/IDS (Image Dissector Scanner) database of $\mathrm{Mg}_{2}$ indices for 381 galaxies and 38 globulars was published by Trager et al. (1998 - hereafter Lick98). This dataset should be regarded as the last version of the Lick/IDS line-strength index system, and we re-scaled all the HYPERCAT data to it.

The estimate of the external errors, and of the corrections to the Lick98 system is done iteratively, and globally for all the HYPERCAT datasets, by inter-comparing these datasets. The comparisons are performed on the aperturecorrected data. For present datasets, the significant intersections are with the LICK dataset (Davies et al. 1987) and with Lick98. 
Table 2. Internal and external comparisons. The first column lists our datasets (with the total number of observed spectra in brackets), Cols. $2-8$ show the internal comparisons between these datasets. Columns 9,10 and 11 show the comparisons (ours - literature) between the datasets of Col. 1 and, respectively, Lick98, LICK, and HYP (for HYPERCAT). The first row of each cell is the number of measurements compared. The second is the mean difference: (line) - (Col.), and the third is the rms. The diagonal (in italics) shows the comparison between consecutive observations of the same galaxy

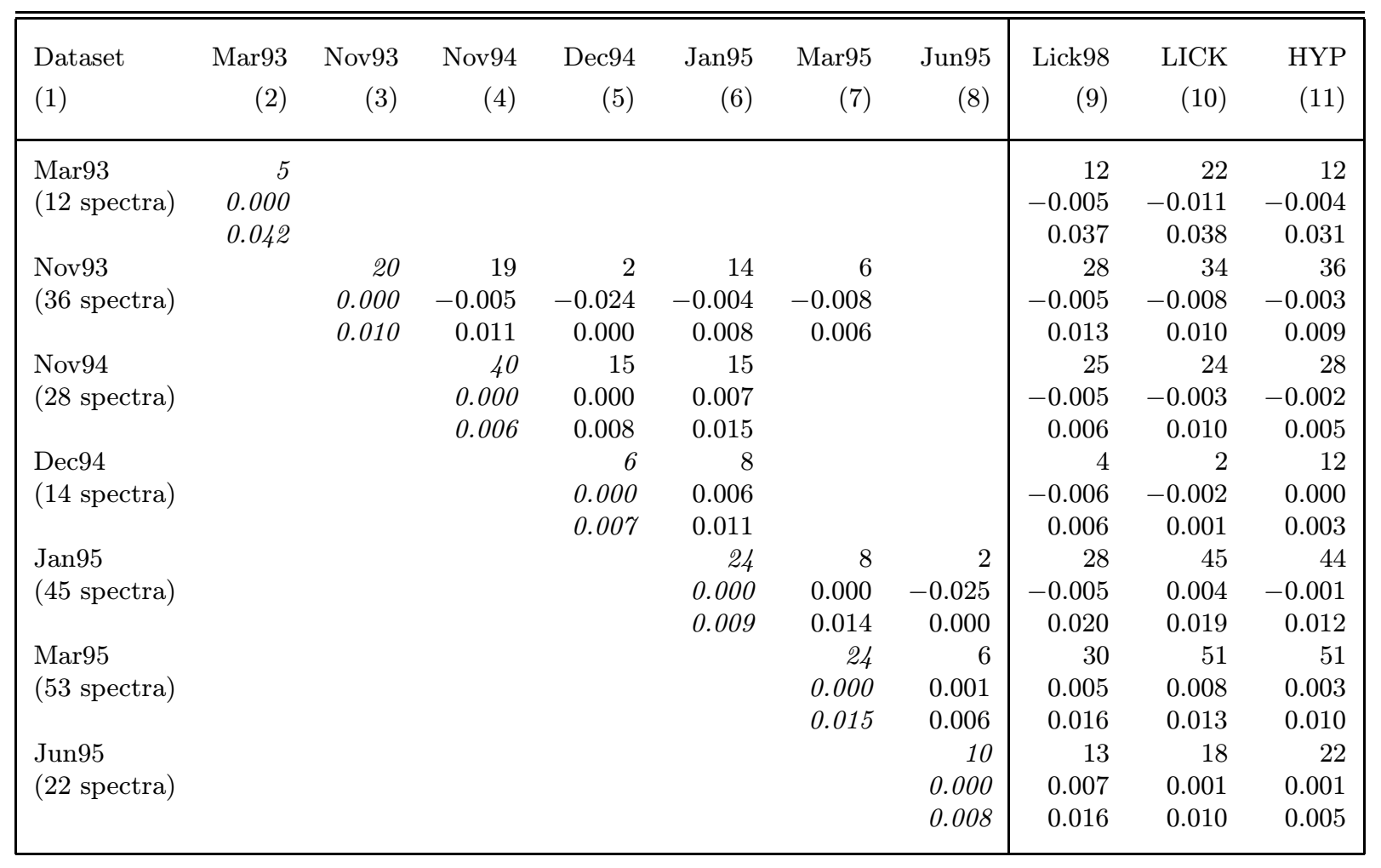

After these comparisons, for 59 galaxies in common with other datasets a difference of $\mathrm{Mg}_{2}$ (our) $\mathrm{Mg}_{2}($ HYPERCAT $)=0 \cdot 018 \pm 0 \cdot 002$ was found. Such difference between flux-calibrated measurements, like ours, and the Lick/IDS data which are normalized via quartz flat field are indeed not surprising. Analysing the details of the pair-comparisons, we adopted a correction of 0.019 for all datasets except Mar93 (0.025) and Mar95 (0.016).

The parameters of our datasets are given in Table 3 (available in electronic form only).

We summarize in Table 2 the final comparisons between the LICK and Lick98 datasets, our datasets and the HYPERCAT homogenized data. The residual zeropoints are negligible, and the mean differences give estimates of the internal errors (on the diagonal of the table are comparisons between consecutive observations; on the other positions are comparisons between different observing runs), and external errors (last three columns). The comparison between our fully corrected measurements and HYPERCAT values is presented in Figs. 1 and 2 (the distributions of residuals with the two major datasets of Table 2 have basically the same form).

Note that the mean residuals between the fully corrected data and the HYPERCAT values are not strictly null, but simply statistically unsignificant. This is because the homogenisation process (Golev \& Prugniel 1998) rests on pair-wise comparison and iteratively determines zeropoints and weights for all the datasets in HYPERCAT. Such process is not stable and thus is hand-controlled at each iteration (this control consists in under-relaxing the corrections to damp the oscillations).

We also searched for correlations between the mean difference of our $\mathrm{Mg}_{2}$-index measurements and any of these datasets (as some non-linearity in the whole acquisition chain could produce). We did not find any significant effect.

In Table 4 we present the mean corrected value of $\mathrm{Mg}_{2}$ rescaled to the Lick system and the corresponding rms error for the 87 galaxies in the sample. In the electronic version of the table more information for each galaxy is provided, e.g. the flow-smoothed velocity used for aperture correction (defined as the velocity of the cosmologic flow associated with the galaxy).

\section{Conclusion}

We have presented 210 new central $\mathrm{Mg}_{2}$ measurements for 87 galaxies rescaled to the Lick/IDS system. 


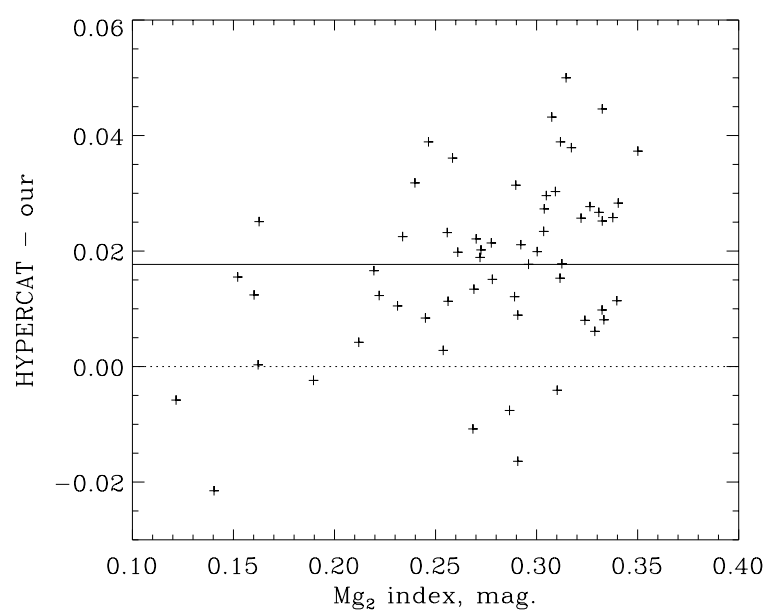

Fig. 1. Comparison between our fully corrected $\mathrm{Mg}_{2}$-index measurements and HYPERCAT values for 59 galaxies in common

The mean individual internal error on these measurements is $0.009 \pm 0.003$ and the external error is $0.012 \pm 0.002$.

Beside providing $\mathrm{Mg}_{2}$ measurements for 28 new galaxies, this analysis shows the suitability of our spectra for line-strength measurements. We will extend this work by measuring the spatial profiles of line strength indices along the major axis of galaxies, and use this data to go further into the analysis and interpretation of the scaling relations involving the stellar population. Two preliminary reports are published in Prugniel \& Golev (1998) and Golev et al. (1998).

Acknowledgements. We are grateful to the telescope operators at Observatoire de Haute-Provence for their help during the observations. This research has made use of the Lyon-Meudon Extragalactic Database (LEDA) operated by the LEDA team at the CRAL-Observatoire de Lyon. This work has benefited from the SIMBAD database operated by the Centre des Données astronomiques de Strasbourg (CDS) in Strasbourg. We acknowledge the use of the ADS Abstract Service. We are grateful to the anonymous referee for useful comments. VG thanks the CRALObservatoire Astronomique de Lyon for an invited-astronomer position and the Bulgarian National Science Fundation grant No. F575/1995.

\section{References}

Busarello G., Capaccioli M., d'Onofrio M., Longo G., Richter G., Zaggia S., 1996, A\&A 314, 32

Cardiel N., Gorgas J., Cenarro J., González J.J., 1998, A\&AS 127,597

de Carvalho R.R., Djorgovski S.G., 1989, ApJ 341, L37

Davies R.L., Burstein D., Dressler A., Faber S.M., Lynden-Bell D., Terlevich R.J., Wegner G., 1987, ApJS 64, 581

Djorgovski S.G., Davies M., 1987, ApJ 313, 59

Djorgovski S.G., Santiago B.X., 1993, in: ESO/EIPC Workshop on Structure, Dynamics and Chemical Evolution

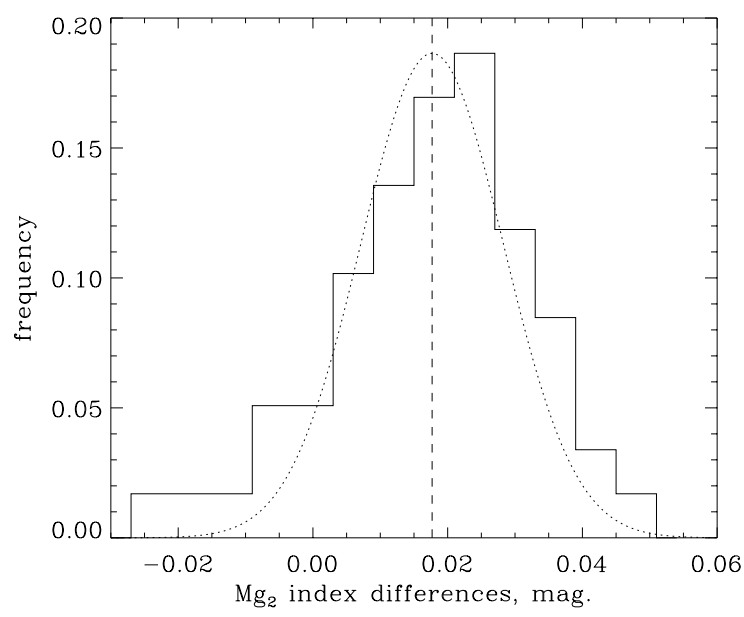

Fig. 2. The distribution of the differences between our $\mathrm{Mg}_{2}-$ index measurements and HYPERCAT values

of Early-type Galaxies, Danziger I.J., Zeilinger W.W., Kjär K., ESO, Garching, p. 59

Dressler A., Lynden-Bell D., Burstein D., Davies R.L., Faber S.M., Terlevich R., Wegner G., 1987, ApJ 313, 42

Faber S.M., Wegner G., Burstein D., Davies R.L., Dressler A., Lynden-Bell D., Terlevich R.J., 1989, ApJS 69, 763 (7Sam) Golev V., Prugniel Ph., 1998, A\&AS 132, 255

Golev V., Prugniel Ph., Simien F., 1998, in: Star Formation in Early-type Galaxies, Cepa J., Carral P., Guanajuato, ASP Conf. Ser. (in press)

Gregg M., 1992, AJ 384, 43

Guzmán R., Lucey J.R., Carter D., Terlevich R.J., 1992, MNRAS 257, 187

Jørgensen I., Franx M., Kjœrgaard P., 1995, MNRAS 276, 1341

Jørgensen I., Franx M., Kjœrgaard P., 1996, MNRAS 280, 167

Leitherer C., Alloin D., Fritze-v. Alvensleben U., et al., 1996, PASP 108, 996

Longhetti M., Rampazzo R., Bressan A., Chiosi C., 1998, A\&AS 130, 251

Prugniel Ph., Golev V., 1998, in: Star Formation in Early-type Galaxies, Cepa J., Carral P., Guanajuato (eds.). ASP Conf. Ser. (in press)

Prugniel Ph., Rampazzo R., Simien F., 1992, La lettre de l'OHP, No. 8, p. 1

Prugniel Ph., Simien F., 1994, A\&A 282, L1

Prugniel Ph., Simien F., 1996, A\&A 309, 749

Prugniel Ph., Simien F., 1997, A\&A 321, 111

Recillas-Cruz E., Serrano P.G.A., Cruz-Gonzàlez I., Carrasco L., 1990, A\&A 229, 64

Renzini A., Ciotti L., 1993, ApJ 416, L49

Scodeggio M., Gavazzi G., Belsole E., Pierini D., Bosseli A., 1998, MNRAS 301, 1001

Simien F., Prugniel Ph., 1997a, A\&AS 112, 521

Simien F., Prugniel Ph., 1997b, A\&AS 126, 15

Simien F., Prugniel Ph., 1997c, A\&AS 126, 519

Simien F., Prugniel Ph., 1998, A\&AS 131, 287

Trager S.C., Worthey G., Faber, S.M., Burstein D., González J.J., 1998, ApJS 116, 1 (Lick98)

Worthey G., Faber S.M., González J.J., Burstein D., 1994, ApJS 94, 687

Worthey G., Ottaviani D.L., 1997, ApJS 111, 377 
Table 4. Fully corrected values of $\mathrm{Mg}_{2}$ indices, rescaled to the Lick system. The galaxy's identifiers are listed in first column. Columns 2 and 3 contain corrected $\mathrm{Mg}_{2}$-index values and the corresponding rms errors. In Col. $4 N$ are the numbers of individual spectra used

\begin{tabular}{cccc}
\hline \hline Identifier & $\mathrm{Mg}_{2}$ (corr) & rms error & $N$ \\
$(1)$ & $(2)$ & $(3)$ & $(4)$ \\
\hline NGC 0097 & 0.277 & 0.008 & 2 \\
NGC 0383 & 0.325 & 0.008 & 2 \\
NGC 0430 & 0.300 & 0.009 & 2 \\
NGC 0499 & 0.332 & 0.004 & 4 \\
NGC 0584 & 0.258 & 0.007 & 2 \\
NGC 0680 & 0.268 & 0.005 & 3 \\
NGC 0855 & 0.050 & 0.009 & 2 \\
NGC 0890 & 0.257 & 0.004 & 4 \\
NGC 0990 & 0.256 & 0.006 & 2 \\
NGC 1016 & 0.319 & 0.007 & 2 \\
NGC 1023 & 0.286 & 0.003 & 6 \\
NGC 1060 & 0.331 & 0.017 & 1 \\
NGC 1132 & 0.275 & 0.014 & 2 \\
NGC 1175 & 0.308 & 0.005 & 2 \\
NGC 1199 & 0.293 & 0.006 & 2 \\
NGC 1209 & 0.285 & 0.008 & 1 \\
NGC 1426 & 0.231 & 0.012 & 2 \\
NGC 1461 & 0.282 & 0.006 & 2 \\
NGC 1521 & 0.263 & 0.007 & 3 \\
NGC 1573 & 0.321 & 0.010 & 2 \\
NGC 1653 & 0.288 & 0.005 & 4 \\
NGC 1684 & 0.281 & 0.014 & 1 \\
NGC 1713 & 0.297 & 0.010 & 2 \\
NGC 1726 & 0.283 & 0.005 & 5 \\
NGC 2314 & 0.310 & 0.008 & 2 \\
NGC 2329 & 0.286 & 0.005 & 4 \\
NGC 2332 & 0.301 & 0.008 & 3 \\
NGC 2340 & 0.321 & 0.008 & 2 \\
NGC 2549 & 0.257 & 0.003 & 8 \\
NGC 2563 & 0.328 & 0.007 & 2 \\
NGC 2685 & 0.215 & 0.004 & 5 \\
NGC 2695 & 0.299 & 0.006 & 2 \\
NGC 2732 & 0.234 & 0.003 & 6 \\
NGC 2768 & 0.261 & 0.003 & 8 \\
NGC 2986 & 0.309 & 0.006 & 2 \\
NGC 3158 & 0.344 & 0.006 & 2 \\
NGC 3193 & 0.264 & 0.010 & 1 \\
\hline & & &
\end{tabular}

Table 4. continued

\begin{tabular}{|c|c|c|c|}
\hline $\begin{array}{l}\text { Identifier } \\
\text { (1) }\end{array}$ & $\begin{array}{c}\mathrm{Mg}_{2}(\text { corr }) \\
(2)\end{array}$ & $\begin{array}{c}\text { rms error } \\
(3)\end{array}$ & $\begin{array}{l}N \\
(4)\end{array}$ \\
\hline NGC 3370 & 0.122 & 0.006 & 2 \\
\hline NGC 3818 & 0.276 & 0.011 & 1 \\
\hline NGC 3853 & 0.280 & 0.007 & 2 \\
\hline NGC 3862 & 0.313 & 0.007 & 2 \\
\hline NGC 3893 & 0.122 & 0.009 & 1 \\
\hline NGC 3921 & 0.134 & 0.006 & 2 \\
\hline NGC 4239 & 0.140 & 0.013 & 2 \\
\hline NGC 4270 & 0.230 & 0.004 & 2 \\
\hline NGC 4283 & 0.259 & 0.011 & 1 \\
\hline NGC 4318 & 0.186 & 0.006 & 2 \\
\hline NGC 4339 & 0.249 & 0.006 & 2 \\
\hline NGC 4340 & 0.243 & 0.006 & 2 \\
\hline NGC 4429 & 0.271 & 0.005 & 2 \\
\hline NGC 4434 & 0.242 & 0.004 & 3 \\
\hline NGC 4435 & 0.228 & 0.005 & 2 \\
\hline NGC 4442 & 0.291 & 0.004 & 2 \\
\hline NGC 4458 & 0.216 & 0.006 & 2 \\
\hline NGC 4461 & 0.277 & 0.005 & 2 \\
\hline NGC 4464 & 0.225 & 0.005 & 2 \\
\hline NGC 4468 & 0.166 & 0.013 & 2 \\
\hline NGC 4476 & 0.145 & 0.007 & 2 \\
\hline NGC 4478 & 0.244 & 0.004 & 4 \\
\hline NGC 4479 & 0.219 & 0.011 & 3 \\
\hline NGC 4489 & 0.193 & 0.010 & 2 \\
\hline NGC 4552 & 0.319 & 0.008 & 1 \\
\hline NGC 4636 & 0.249 & 0.030 & 2 \\
\hline NGC 4638 & 0.256 & 0.010 & 1 \\
\hline NGC 4874 & 0.310 & 0.011 & 2 \\
\hline NGC 4886 & 0.247 & 0.011 & 2 \\
\hline NGC 5129 & 0.279 & 0.011 & 1 \\
\hline NGC 5353 & 0.327 & 0.004 & 2 \\
\hline NGC 5424 & 0.285 & 0.005 & 4 \\
\hline NGC 5490 & 0.330 & 0.005 & 3 \\
\hline NGC 5557 & 0.299 & 0.004 & 2 \\
\hline NGC 5854 & 0.171 & 0.007 & 2 \\
\hline NGC 5864 & 0.217 & 0.009 & 1 \\
\hline NGC 5966 & 0.260 & 0.006 & 2 \\
\hline NGC 6137 & 0.329 & 0.017 & 2 \\
\hline NGC 6146 & 0.287 & 0.004 & 2 \\
\hline NGC 6166 & 0.307 & 0.028 & 1 \\
\hline NGC 7177 & 0.188 & 0.009 & 1 \\
\hline NGC 7331 & 0.218 & 0.007 & 1 \\
\hline NGC 7332 & 0.218 & 0.004 & 4 \\
\hline NGC 7457 & 0.159 & 0.005 & 3 \\
\hline NGC 7660 & 0.321 & 0.008 & 2 \\
\hline NGC 7727 & 0.284 & 0.006 & 2 \\
\hline NGC 7778 & 0.286 & 0.009 & 2 \\
\hline NGC 7785 & 0.281 & 0.007 & 2 \\
\hline UGC 03696 & 0.293 & 0.005 & 3 \\
\hline UGC 03792 & 0.228 & 0.018 & 2 \\
\hline
\end{tabular}

